\title{
Sobre los episodios micropsicóticos y el sentimiento de vacío.
}

\author{
Regarding micro-psychotic episodes and chronic feelings of emptiness.
}

\author{
Alonso Garrido-Pinzás 1,2,a
}

\section{Señores Editores:}

Clásicamente un paciente con personalidad limítrofe no debería presentar sintomatología psicótica, sin embargo en el tiempo se ha ido aceptando la aparición breve de alucinaciones o delusiones, hecho que desdibuja la línea diagnóstica entre estos pacientes y los francamente psicóticos (1). Podemos entender que al referirnos a estos términos debemos ahondar un poco más en las características, ya que al tildar o etiquetar a un paciente de "borderline" por la presencia de estos síntomas corremos el riesgo de hacer un diagnóstico equivocado.

El Manual Diagnóstico y Estadístico de la Asociación Psiquiátrica Americana (DSM-5), incluye dentro de su lista de criterios diagnósticos del trastorno de personalidad limítrofe la sensación crónica de vacío, y las ideas paranoides transitorias relacionadas con el estrés o síntomas disociativos graves ("episodios micropsicóticos"). Estas características han sido estudiadas previamente y no siempre exclusivamente en el trastorno limítrofe de personalidad (TLP). En la práctica clínica es frecuente que se mencionen y se discutan estos dos elementos, a pesar de que no existe un consenso con respecto a su definición. Entre 1-2\% de la población general $(2,3), 10-12 \%$ de la población de pacientes psiquiátricos ambulatorios, $20-22 \%$ de los pacientes psiquiátricos hospitalizados presenta TLP (3), y se estima que un $20-50 \%$ de estos pacientes presenta síntomas psicóticos (4). Por ello consideramos que es necesaria una revisión al respecto.

Sensación crónica de vacio: El sentimiento de vacío es difícil de describir; es un concepto artístico, filosófico, teológico, y psicológico (5). Algunos pacientes pueden definir el sentimiento de vacío como una experiencia somática ("me siento vacio por dentro") y otros como un "vacío existencial" o una "falta de sentido" (6). Asimismo, el sentimiento de vacío no es exclusivo del trastorno de personalidad limítrofe; se describe en pacientes con trastornos psicóticos y depresivos, así como en pacientes con otros trastornos de personalidad. Los pacientes narcisistas pueden describir un sentimiento de vacío resultante del aburrimiento y la falta de gratificación al interactuar con otras personas, y los pacientes esquizoides pueden describir lo mismo pero esta vez por una característica innata que hace que se sientan diferentes a los demás, distantes, e incapaces de sentir $(7,8)$.

Un estudio demostró que el sentimiento de vacío está estrechamente relacionado con sentimientos de desesperanza, soledad, y aislamiento, además de estar relacionado a la depresión y ser -después del criterio relacionado con conductas suicidas o de

Universidad Peruana Cayetano Heredia. Lima, Perú.

Hospital Nacional Cayetano Heredia. Lima, Perú.

Médico residente de Psiquiatría ; ORCID ID: 0000-0002-9216-1017 
automutilación- el criterio más asociado a la ideación suicida (9). Un análisis de redes (network analysis) de características psicopatológicas del año 2018 encontró que la característica central era la sensación crónica de vacío (chronic emptiness) (10). Por otro lado, en una revisión sistemática del año 2020 que buscaba definir y entender el sentimiento crónico de vacío se evaluó 99 estudios al respecto, y concluyó que no existía una definición clara y consecuentemente evidenció las dificultades para cuantificarlo (11).

Episodios micropsicóticos: En la práctica clínica se suele utilizar el término "episodios micropsicóticos" (o cuasi psicóticos), para referirse a episodios psicóticos transitorios que emergen en situaciones de estrés $(2,4,8)$. Sin embargo, el término fue usado por primera vez por Hoch y Polatin en 1949 (1), en un artículo en el que describen las características de la llamada Esquizofrenia Pseudoneurótica, en que los pacientes "van en zig-zag sobre la línea de la realidad": episodios psicóticos cortos con recuperación tan completa al punto de que "si es que uno no fue testigo de sus episodios psicóticos, no creería que estuvieron psicóticos" (12). Además, estos episodios eran caracterizados por tres elementos importantes, muchas veces entrelazados: ideas hipocondriacas, ideas de referencia, y sentimientos de despersonalización (12). Koldobsky agrega que estos episodios pueden también incluir desorganización cognitiva, y que la frecuencia usualmente disminuye con el tiempo (13). Kety, Rosenthai and Wender (14), al describir las características de la "esquizofrenia limítrofe" resaltaron también la existencia de experiencias de despersonalización y desrealización.

A pesar de que no hemos encontrado una definición concisa, podemos resaltar algunos elementos comunes a través de las diferentes fuentes; los episodios micropsicóticos: 1) son transitorios, 2) aparecen como respuesta a situaciones estresantes en personas con personalidades predispuestas, 3) pueden solucionarse sin tratamiento, y 4) tienen recuperación completa. No encontramos un rango de duración establecido, y consideramos la posibilidad de la conciencia pueda estar comprometida (ya que la despersonalización y la desrealización son de carácter histeriforme y presuponen alteraciones de la conciencia).

Luego de esta pequeña revisión concluimos que existen múltiples definiciones y formas de conceptualizar el sentimiento de vacío, y que por otro lado no existe una definición clara y concisa de lo que representa el término "micropsicosis".
Asimismo, se hace evidente la falta de estudios de carácter psicopatológico y clínico sobre estos temas y la necesidad de entender plenamente estos conceptos para poder establecer en consenso definiciones claras a ser utilizadas en la práctica clínica. Creemos que ambas dudas se podrían esclarecer inicialmente con estudios cualitativos que servirían de punto de partida para estudios más rigurosos y de mayor escala.

Agradecimientos: a los doctores Patricia Albornoz, Lizardo Cruzado, Martín Arévalo, Alfredo Valencia, Cynthia Cabrejos, y Lucía Luna

Conflictos de intereses: Ninguno

Financiamiento: Autofinanciado

\section{Correspondencia}

Alonso Garrido-Pinzás

Av Juan Antonio Pezet 1997, dpto 402, San Isidro Lima 15076, Perú

Correo electrónico: alonso.garrido@upch.pe

\section{REFERENCIAS BIBLIOGRÁFICAS}

1. Waters F, Stephane M. The Assessment of Psychosis. A reference book and rating scales for research and practice. 1st ed. New York: Routledge; 2015.

2. Kaplan H, Sadock V, Sadock B. Kaplan \& Sadock's comprehensive textbook of psychiatry. Philadelphia, Pennsylvania: Lippincott Williams \& Wilkins; 2005.

3. Ellison W, Rosenstein L, Morgan T, Zimmerman M. Community and Clinical Epidemiology of Borderline Personality Disorder. Psychiatr Clin North Am. 2018; 41(4):561-573.

4. Schultz HE, Hong V. Psychosis in borderline personality disorder: how assessment and treatment differs from a psychotic disorder. Curr Psychiatry. 2017; 16:24-29.

5. Hazell C. A scale for measuring experienced levels of emptiness and existential concern. The Journal of Psychology. 1984; 117(2):177-182.

6. Elsner D, Broadbear J, Rao S. What is the clinical significance of chronic emptiness in borderline personality disorder? Australas Psychiatry. 2017;26(1):88-91.

7. Zandersen M, Parnas J. Identity Disturbance, feelings of emptiness, and the boundaries of the schizophrenia spectrum. Schizophr Bull. 2018;45(1):106-113.

8. Kernberg O. Borderline conditions and pathological narcissism. Lanham: Rowman \& Littlefield; 2004.

9. Klonsky E. What is Emptiness? Clarifying the 7th Criterion for Borderline Personality Disorder. J Pers Disord. 2008;22(4):418-426

10. Southward, MW, Cheavens, JS. Identifying core 
Sobre los episodios micropsicóticos y el sentimiento de vacío.

deficitsinadimensionalmodelofborderlinepersonality disorder features: A network analysis. Clin Psychol Sci. 2018; 6(5):685-703 doi:10.1177/21677026 18769560

11. Miller CE, Townsend ML, Day NJS, Grenyer BFS. Measuring the shadows: A systematic review of chronic emptiness in borderline personality disorder. PLoS ONE. 2020; 15(7): e0233970. DOI: 10.1371/ journal.pone. 0233970
12. Hoch P, Polatin P. Pseudoneurotic forms of schizophrenia. Psychiatr Q. 1949;23(2):248-276.

13. Koldobsky N. Trastorno borderline de la personalidad. Buenos Aires: Editorial Polemos; 2005.

14. Rothenhäusler H, Kapfhammer, H. Der Verlauf von Borderline- Störungen. Fortschritte Der Neurologie Psychiatrie. 1999; 67(05), 200-217. doi:10.1055/s-2007-993997 